putative promoter in intron 7 of USF1. Although the usf1s2 polymorphism, which resides in or close to the putative promoter, had no impact on transcriptional activity, its identification is somewhat encouraging. In principle, the putative promoter could enable USF1 to initiate translation from one of two internal AUGs in exon 8 and, in doing so, throw a switch that reverses the normal function of this protein from activation to repression or vice versa, as has been described for a number of other transcription factors ${ }^{12}$ (Fig. 1). Evidence that the intron 7 promoter is used in vivo will be keenly awaited.

In summary, the work of Pajukanta et al. ${ }^{2}$ has identified USF1 as the prime candidate in the chromosome 1q21-23 linkage region for increasing susceptibility to FCHL, type 2 diabetes and metabolic syndrome. The challenges ahead will be to confirm the genetic data through replication, to identify other genes associated with FCHL at additional loci and to determine the roles of the different isoforms of USF1 in the regulation of genes pivotal for whole-body lipid and glucose homeostasis and the maintenance of the arterial wall vasculature ${ }^{13}$.

1. Lewis, G.F. Curr. Opin. Lipidol. 13, 97-99 (2002).

2. Pajukanta, P. et al. Nat. Genet. 36, 371-376 (2004)

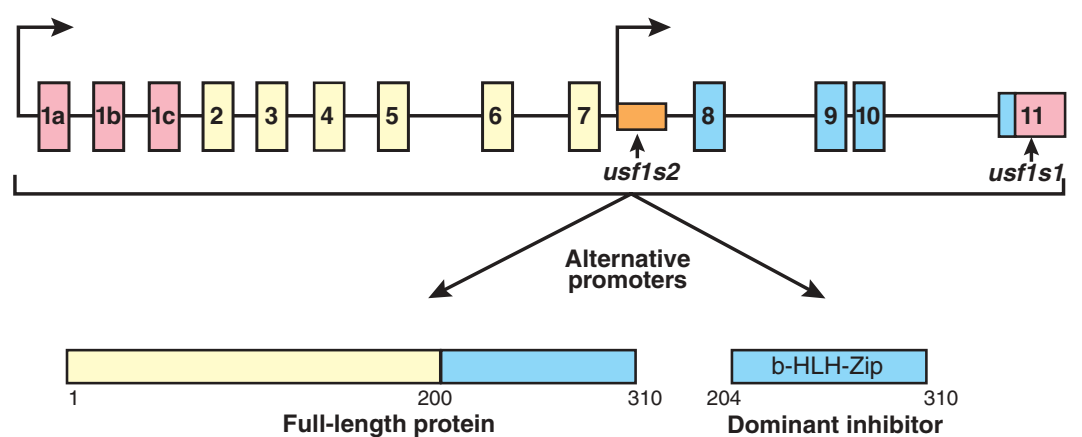

Figure 1 A theoretical scenario to illustrate how the use of a putative promoter in intron 7 of USF1 (orange box) might lead to the generation of a mini-USF1 protein lacking the transactivation domain. Because mini-USF proteins in vitro behave as transdominant inhibitors ${ }^{14,15}$, there is a substantial incentive to establish whether the putative promoter identified by Pajukanta et l. $^{2}$ might operate in vivo to downregulate USF1 activity. The two polymorphisms associated with FCHL in Finnish families are indicated. b-HLH-Zip, basic helix-loop-helix-leucine zipper.

3. Pajukanta, P. et al. Nat. Genet. 18, 369-373 (1998).

4. Pei, L. J. Biol. Chem. 276, 8484-8491 (2001).

5. Coon, H. et al. Arterioscler. Thromb. Vasc. Biol. 20, 2275-2280 (2000).

6. McCarthy, M.I. Curr. Diab. Rep. 3, 159-167 (2003).

7. Smih, F. et al. Diabetes 51, 293-300 (2002).

8. Moore, M.L., Park, E.A. \& McMillin, J.B. J. Biol. Chem. 278, 17263-17268 (2003).

9. Risch, N. \& Merikangas, K. Science 273, 1516-1517 (1996)

10. Page, G.P., George, V., Go, R.C., Page, P.Z. \& Allison, D.B. Am. J. Hum. Genet. 73, 711-719 (2003).
11. Shoulders, C.C., Jones, E.L. \& Naoumova, R.P. Hum. Mol. Genet. 13, R149-R160 (2004).

12. Foulkes, N.S. \& Sassone-Corsi, P. Cel/ 68, 411-414 (1992).

13. Chen, Y.H., Layne, M.D., Watanabe, M., Yet, S.F. \& Perrella, M.A. J. Biol. Chem. 276, 47658-47663 (2001).

14. Lefrancois-Martinez, A.M., Martinez, A., Antoine, B., Raymondjean, M. \& Kahn, A. J. Biol. Chem. 270, 2640-2643 (1995).

15. Viollet, B. et al. J. Biol. Chem. 271, 1405-1415 (1996)

\title{
Phenotype matters
}

\author{
Leslie G Biesecker
}

\section{The association of diseases with genes is complex, even among mendelian disorders. A new study shows that mutations in the gene encoding filamin B (FLNB) cause four distinct disorders of human skeletal development.}

\begin{abstract}
Mapping a mendelian disorder to a genetic locus is easier than determining the relationships between genes and disorders or between different disorders. Initially, it seemed that the correspondence of diseases to genes would be simple: one gene, one disease. As we have learned from positional cloning efforts, this is often untrue. But, as a new study by Deborah Krakow and colleagues ${ }^{1}$ illustrates, the reality is far more interesting.

They found that mutations in FLNB, encoding filamin $\mathrm{B}$, which belongs to a family of proteins that act as key regulators of the actin cytoskeleton, underlie four distinct

Leslie G. Biesecker is at the National Human Genome Research Institute, National Institutes of Health, Bethesda, Maryland. e-mail: leslieb@helix.nih.gov
\end{abstract}

human skeletal disorders ${ }^{1}$. The implications of this work are many, and two in particular deserve attention.

\section{Different vantage points}

From a clinical genetics standpoint, spondylocarpotarsal syndrome (OMIM 272460), Larsen syndrome (OMIM 150250) and atelosteogenesis types I (AOI; OMIM 108720) and III (AOIII; OMIM 108721) are four distinct human disorders. In contrast, a basic scientist may view them as a single disorder of filamin B dysfunction with inconsequential phenotypic differences. Both perspectives are valid, but they may result in incompatible classification schemes that impede a comprehensive understanding of human genetic disease.

The determination of allelism does not supplant phenotypic distinctions. Although AOI and AOIII share some features, the phenotypic distinctions are important. The qualitative differences in phenotypic manifestations that distinguish atelosteogenesis from spondylocarpotarsal and Larsen syndromes tell us something about filamin B biology. This information follows on the heels of a report describing mutations in a related gene, FLNA, as the cause of five distinct disorders in humans $^{2}$ (otopalatodigital syndromes types I and II, OMIM 311300 and 304120, respectively; Melnick-Needles syndrome, OMIM 309350; periventricular nodular heterotopia, OMIM 300049; and frontometaphyseal dysplasia, OMIM 305620). This is interesting because there are overlapping phenotypic features in the disorders associated with FLNA and FLNB (Fig. 1). Furthermore, Larsen syndrome probably has genetic heterogeneity, as 


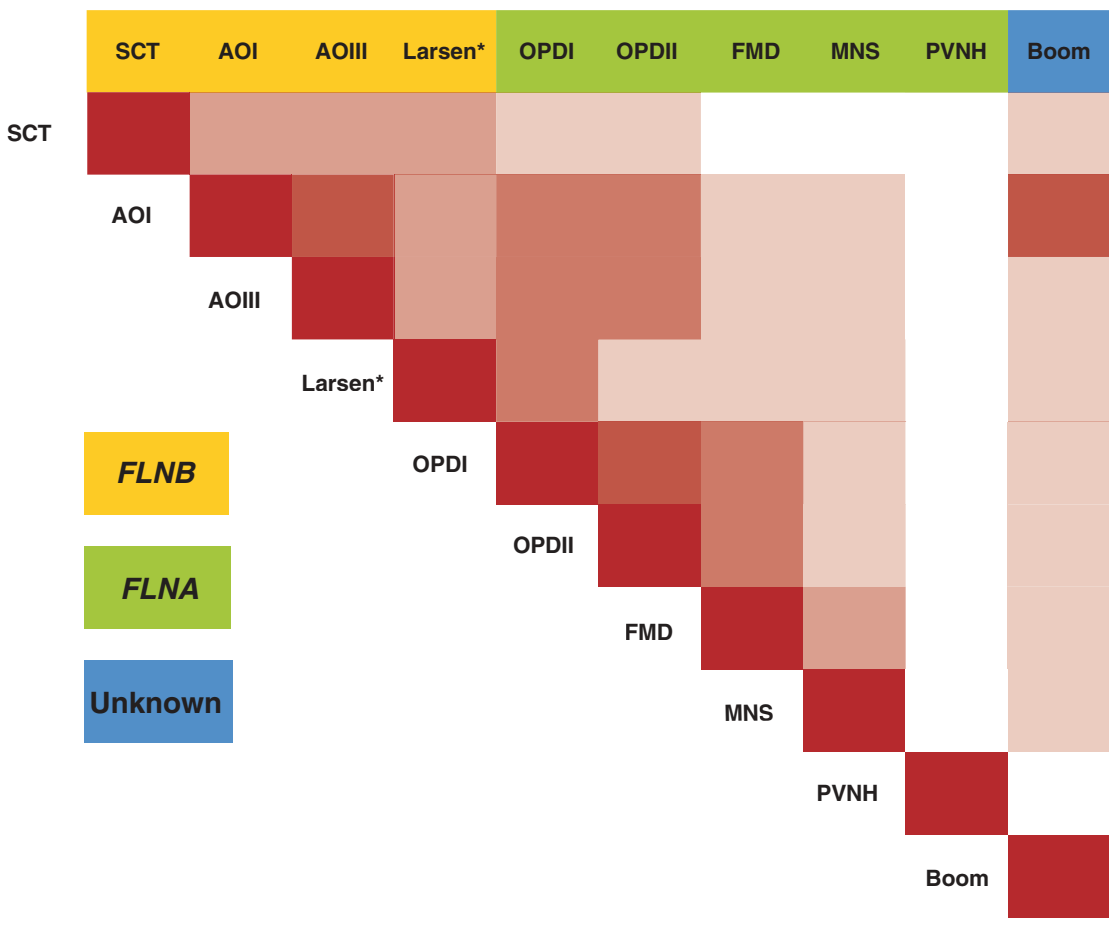

Figure 1 The overall similarity of the various disorders associated with mutations in FLNA or in FLNB and of disorders with unknown genetic etiology based on information from several sources ${ }^{4-6}$. Darker shading indicates greater phenotypic similarity of disorders. SCT, spondylocarpotarsal syndrome; OPDI and OPDII, otopalatodigital syndrome types I and II, respectively; FMD, frontometaphyseal dysplasia; MNS, Melnick-Needles syndrome; PVNH, periventricular nodular heterotopia; Boom, Boomerang dysplasia (OMIM 112310). The asterisk indicates that the phenotype of Larsen syndrome probably manifests locus heterogeneity.

there is an apparent autosomal recessive form of Larsen syndrome as well. This does not mean, however, that there are two clinical types of Larsen syndrome, as we do not know whether individuals with Larsen syndrome associated or not associated with FLNB are phenotypically distinct.

One implication of the destruction of the one gene-one disease model is that we need to develop ways to describe and code affected individuals, both in the clinic and in research studies, that take into account this heterogeneity $^{3}$. A second implication is that we need to redouble our efforts to understand the relationships of phenotypes to genotypes, to gain biological insight into gene function and to optimally manage and counsel affected individuals. useful for medical care and estimating recurrence risks) and, as in the FLNB story, contribute to research. Whereas a lone investigator working on one or a few phenotypes would be much less likely to uncover the etiology of these disorders, the intersection of sharp clinical skills, an extensive archive of cases and the power of positional cloning resulted in discovery.

This is the future of human genetic research. As the low-hanging fruit has been picked, only the rarest and genetically more complex disorders remain, and these cannot be efficiently studied by single investigators working with small, narrowly defined groups of affected individuals. More resources like ISDR need to be assembled, and those resources need to be widely available. Scientists of many disciplines need to be encouraged to draw on the expertise, archives and tissues of the registries to accelerate scientific discovery. In addition, those who invest the enormous resources necessary to build such registries must be supported financially and academically, including academic credit and promotions. The creation and appropriate use of such registries is difficult, which is why there are so few of them. Yet if we wish for these advances to continue, registries like ISDR must be developed and expanded.

The filamin story is just beginning to be unraveled, but it has already provided several useful lessons about the relationships of genes to diseases and the resources necessary to uncover those relationships. No doubt there will be more surprising discoveries about the biology of this system and the relationships of these gene products to human skeletal development.

1. Krakow, D. et al. Nat. Genet. 36, 405-410 (2004).

2. Robertson, S.P. et al. Nat. Genet. 33, 487-491 (2003).

3. Robin, N.H. \& Biesecker, L.G. Genet. Med. 3, 290-293 (2001).

4. Taybi, H. \& Lachman, R.S. Radiology of Syndromes, Metabolic Disorders, and Skeletal Dysplasias (Mosby, St. Louis, 1996).

5. Jones, K.L. Smith's Recognizable Patterns of Human Malformation (W B Saunders, Philadelphia, 1997).

6. Spranger, J.W., Brill, P.W. \& Poznanski, A.K. Bone Dysplasias: An Atlas of Genetic Disorders of Skeletal Development. (Oxford University Press, Oxford, 2002). 